\title{
Qi-Shen-Yi-Qi Dripping Pills for the Secondary Prevention of Myocardial Infarction: A Randomised Clinical Trial
}

\author{
Hongcai Shang, ${ }^{1}$ Junhua Zhang, ${ }^{1}$ Chen Yao, ${ }^{2}$ Baoyan Liu, ${ }^{3}$ Xiumei Gao, ${ }^{1}$ Ming Ren, ${ }^{1}$ \\ Hongbao Cao, ${ }_{1}^{1}$ Guohua Dai, ${ }^{4}$ Weiliang Weng, ${ }^{5}$ Sainan $\mathrm{Zhu}^{2}$ Hui Wang, \\ Hongjuan $\mathrm{Xu},{ }^{1}$ and Boli $\mathrm{Zhang}^{1}$ \\ ${ }^{1}$ Tianjin University of Traditional Chinese Medicine, 312 Anshanxi Road, Nankai District, Tianjin 300193, China \\ ${ }^{2}$ Peking University First Hospital, Beijing 100034, China \\ ${ }^{3}$ Chinese Academy of Traditional Chinese Medicine, Beijing 100700, China \\ ${ }^{4}$ Affiliated Hospital of Shandong University of Traditional Chinese Medicine, Jinan, Shandong 250011, China \\ ${ }^{5}$ Xiyuan Hospital, Beijing 100091, China
}

Correspondence should be addressed to Hongcai Shang; shanghongcai@foxmail.com and Boli Zhang; zhangbolipr@163.com

Received 20 March 2013; Accepted 25 May 2013

Academic Editor: Hao Xu

Copyright (c) 2013 Hongcai Shang et al. This is an open access article distributed under the Creative Commons Attribution License, which permits unrestricted use, distribution, and reproduction in any medium, provided the original work is properly cited.

\begin{abstract}
Background. Several types of drugs have been recommended for the secondary prevention of myocardial infarction (MI). However, these conventional strategies have several limitations, such as low adherence, high cost, and side effects during long time use. Novel approaches to this problem are still needed. This trial aimed to test the effectiveness and safety of Qi-Shen-Yi-Qi Dripping Pills (QSYQ), a multi-ingredient Chinese patent medicine, for the secondary prevention of MI. Methods and Findings. A total of 3505 eligible patients were randomly assigned to QSYQ group (1746 patients) or aspirin group (1759). Patients took their treatments for 12 months. The final follow-up visit took place 6 months after the end of the trial drugs. The 12-month and 18-month estimated incidences of the primary outcome were $2.98 \%$ and 3.67\%, respectively, in the QSYQ group. The figures were $2.96 \%$ and 3.81\% in the aspirin group. No significant difference was identified between the groups. Conclusions. This trial did not show significant difference of primary and secondary outcomes between aspirin and QSYQ in patients who have had an MI. Though inconclusive, the result suggests that QSYQ has similar effects to aspirin in the secondary prevention of MI.
\end{abstract}

\section{Introduction}

Acute myocardial infarction is a leading cause of death worldwide [1]. More than 7 million people a year have a myocardial infarction (MI) [2]. Over the past three decades, MI has emerged from an illness seen predominantly in developed countries to more common in developing countries [2-4]. Progresses in emergency management have led to substantial reductions in the mortality rate of acute MI [2]. However, survivors from acute MI remain at greatly increased risk of serious vascular events $[2,5]$. Thus, secondary prevention aimed to decrease mortality and morbidity in survivors after acute $\mathrm{MI}$ is of great, and increasing, significance around the world.

Platelets play a key role in the development of thrombotic and ischemic diseases [6]. Antiplatelet therapy is a major strategy for treating and preventing MI. Anti-platelet drugs have been shown to have definite and substantial net benefits for people who have occlusive vascular disease [7]. Aspirin is a safety and most cost-effective one of the anti-platelet drugs [8]. Current guidelines recommend low-dose aspirin (75$150 \mathrm{mg}$ daily) for the secondary prevention of MI in many countries. However, there are several limitations related to this drug. Long-term therapy with aspirin is associated with an increase in the incidence of symptomatic peptic ulcer, duodenal ulcers, and gastrointestinal and intracranial haemorrhage, even when used at low doses or in buffered or entericcoated formulations $[9,10]$. In a population-based cohort with 4.1 million citizens in Italy, aspirin increased the risk of major gastrointestinal or cerebral bleeding episodes; patients with diabetes had a high rate of bleeding [11]. Furthermore, 


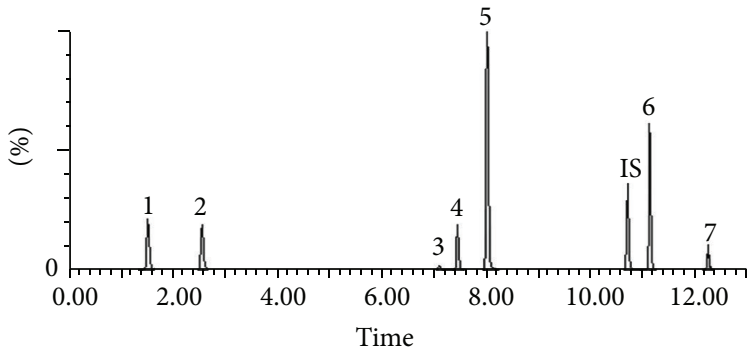

(a)

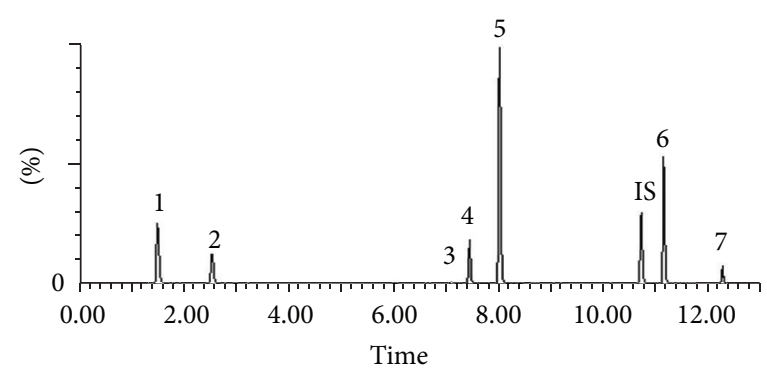

(b)

FIGURE 1: The typical chromatograms of mixed standard solution (a) and sample solution of "Qi-Shen-Yi-Qi” Dripping Pills (b). Numbers 1-7 represent danshensu, protocatechuic-aldehyde, salvianolic acid B, notoginsenoside R1, ginsenoside Rg1, ginsenoside Rb1, and astragaloside IV, respectively. IS represents digoxin. (Redrew for publication).

aspirin resistance has become a notable problem. Several studies have found that about 1 in 4 individuals may express biochemically defined aspirin resistance [12-14]. Patients who are resistant to aspirin are at greater risks of recurrent serious vascular events than those who are sensitive to aspirin $[13,14]$.

Current anti-platelet therapies are generally based on a specific signaling pathway in platelet activation, that is, single agent acting on single target [6]. Hence, the limitations associated with aspirin also exist for other anti-platelet agents, such as clopidogrel [6]. Agents with multipleingredients acting on multiple targets may be more effective and less harmful [15].

In Traditional Chinese Medicine (TCM), the key pathogenesis of MI is mainly "Qixu" (vital energy deficiency) and "Xueyu" (blood stagnation), that is, degradation of body function and thrombosis. Qi-Shen-Yi-Qi Dripping Pills (QSYQ), a Chinese patent medicine for adding "qi" and resolving stasis, was approved for clinical use for coronary heart disease and MI rehabilitation by the State Food and Drug Administration of China in 2003. QSYQ is made of extractions from Danshen (Radix Salviae Miltiorrhizae), Sanqi (radix notoginseng), Jiangxiang (Lignum Dalbergiae Odoriferae), and Huangqi (radix astragali). The quality control of QSYQ is good. Herbal materials were cultivated according to the Good Agriculture Practices, and manufacturing processes strictly followed the standard of Good Manufacturing Practices. UPLC-MS/MS was used to analyze seven quality control markers of QSYQ (Figure 1). There was good consistency of the active markers among different batches [16]. Pharmacological studies revealed that constituents of QSYQ could inhibit the platelet aggregation and the overrelease of $\beta$-TG [17]. Clinical studies have suggested that QSYQ had similar effect to aspirin in inhibiting platelet aggregation [18]. Currently, QSYQ is widely used for the secondary prevention of MI in China. However, there is insufficient evidence to know whether QSYQ can be used as an alternative to aspirin. This multicenter randomized clinical trial aimed to test whether the regular administration of QSYQ would result in a significant reduction in total serious vascular events in patients who had experienced at least one documented MI.

\section{Methods}

2.1. Trial Design. The clinical trial is a multi-center, randomised, double-blind, parallel controlled study. Approval was obtained from the State Administration of Traditional Chinese Medicine of China in 2004. The study protocol was reviewed and approved by the Ethics Committee in Tianjin University of Traditional Chinese Medicine. This trial was registered in the WHO Clinical Trial Registering Platform, number ChiCTR-TRC-00000002 (http://apps.who.int/trialsearch/). The study protocol was summarized here.

2.2. Participates. Patients with a definite diagnosis of STelevation or non-ST-elevation MI [19] were potentially eligible for this study if they met the following criteria: (1) the last documented MI was 4 weeks to 24 months earlier; (2) Traditional Chinese Medicine symptoms were "Qixu-Xueyu” (vital energy deficiency combining with blood stagnation); (3) age from 18 years to 75 years (the maximum age was adjusted from 65 years to 75 years in July 2006 because of inadequate recruitment).

Patients were required to be free of other life-threatening diseases or problems which might have limited the ability to obtain long-time followup and to be free of any condition which would mean that regular use of the trial drugs was contraindicated. Patients with either of the following conditions were excluded: (1) a history of percutaneous coronary intervention (PCI) or coronary artery bypass graft surgery (CABG); (2) pregnant women or those who were breast feeding; (3) contraindication to aspirin (e.g., asthma, active phase peptic ulcer, and hemorrhagic disease); (4) heart function of grade IV (NYHA grade); (5) uncontrolled systemic hypertension (contractive pressure $\geq 180 \mathrm{mmHg}$ or diastolic pressure $\geq 110 \mathrm{mmHg}$ ); (6) uncontrolled serious cardiac arrhythmias (e.g., atrial fibrillation and supraventricular tachycardia); (7) serious primary disease of liver, kidney, and hemopoietic system, or psychosis, or malignant tumor; (8) allergic history to study drugs; (9) participation in other clinical trials during the previous three months.

After a comprehensive medical evaluation, patients were given a full explanation of the study by investigators. Each patient was asked for their written informed consent before joining the study. Patients were registered if they voluntarily 
signed the informed consent. There was no economic compensation for participating patients. Recruitment took place in 88 hospitals throughout the east, west, south, north, and center of China.

2.3. Intervention. Patients were randomly assigned to two groups (ratio 1:1): QSYQ group and aspirin group. In the QSYQ group, patients took $0.5 \mathrm{~g}$ (one package) QSYQ for three times per day and $100 \mathrm{mg}$ (in four tablets) simulated enteric-coated aspirin once a day. Patients in the aspirin group took $0.5 \mathrm{~g}$ (one package) simulated QSYQ three times per day and $100 \mathrm{mg}$ (in four tablets) enteric-coated aspirin daily.

Patients were prohibited from taking other anti-platelet drugs or "Yiqi-Huoxue" Chinese medicines during the treatment period. Concomitant medications, such as antihypertensive (e.g., $\beta$-blockers and ACE inhibitors), hypoglycemic agents, and lipid-lowering drugs, could be prescribed at the discretion of the attending physicians and had to be recorded in detail (including drug name, beginning-stopping time, dosage, and purpose).

The treatment period for the trial drugs was 12 months. After this time (or if the trial drugs were stopped for some other reason), patients could be prescribed treatments by their physicians without any limitation.

2.4. Outcome Measures. The primary endpoint was a composite of cardiovascular death, nonfatal reinfarction (documented by ECG and enzyme changes), and nonfatal stroke (diagnosed by CT or MRI). The secondary outcomes were the events of serious cardiac arrhythmias, heart failure, cardiac shock, revascularization (PCI or CABG), pulmonary embolism, and deep vein thrombosis.

All reported adverse events during the trial were recorded to allow an assessment of the safety of the treatments. Intracranial bleedings and gastrointestinal complications that have been associated with aspirin were monitored carefully. Haemorrhagic stroke was also considered as an adverse event, although it was included in the composite primary outcome.

2.5. Followup. After a first visit for collecting baseline data after randomization, enrolled patients, their dependents, or both were asked to visit clinical centers monthly. If no primary endpoints occurred, there were 12 visits during the treatment period and a final visit ( 6 months after the termination of the trial drugs). When a patient had one of the primary endpoints, the case was considered completed and there was no further follow-up visit. At each visit, investigators were required to retrieve and provide trial drugs; complete case report forms recording the patient's condition, endpoints, adverse events, and concomitant drugs; and remind patients not to take prohibited drugs. If a patient did not visit the clinical center at a defined time, the investigators contacted the patient or their dependents to find the reasons.

2.6. Quality Control Procedures. The trial was designed, executed, and analyzed by a steering committee, a clinical monitoring center, an endpoints committee, a drug management center, a data management center, and a biostatistics center. Investigators in each clinical center were trained before study beginning.

Concealment of the random allocation was achieved by using a Clinical Research Interactive Voice Respond System (CRIVRS). Investigators connected to the CRIVRS by telephone when a patient was ready to be randomised and the CRIVRS then provided a subject number, randomisation code, and drug number to the investigator by voice, email, and SMS. Because different dosage forms of two study drugs, a double-dummy design was used with placebos to blind patients and their healthcare providers. Placebos for QSYQ and aspirin were developed which had the same appearance, color, and taste as the relevant drug. One statistician who generated the blinding code was aware of the drug allocation, but patients, investigators, and other practitioners in this trial were all blinded during the whole study period.

2.7. Statistical Analyses. Due to a paucity of clinical data of QSYQ for the prevention of MI, the sample size for this study was estimated on the basis of practical considerations. The incident of recurred serious cardiovascular and cerebrovascular events was about $6.5 \%$ in patients who suffered from MI and used aspirin for secondary prevention [8]. It was supposed that QSYQ might have equal effectiveness compared with aspirin. The margin of equivalence $(\Delta)$ was $2.5 \%$ for primary endpoint. The sample size of 3600 subjects was calculated to be sufficient to establish equivalence (two sides $\alpha=0.05$ and $80 \%$ power; less than $20 \%$ loss to followup).

Statistical analyses were conducted with SAS 9.1.3. The trial database was blind reviewed before the data were locked and unblinded. Data analyses were mainly based on the intention-to-treat (ITT) principle: with all randomized patients and all endpoints being analysed in accordance with the patients' allocated treatment group. A per-protocol (PP) analysis was performed to test the robust of the trial's results.

The baseline characteristics of the two treatment groups were compared by the $t$-test (for continuous data) and the chisquare test (for categorical data). Cox proportional-hazards regression model was used to estimate the hazard ratio (HR) and $95 \%$ confidence interval (CI) for the primary endpoint. Cumulative primary endpoints curves were constructed by Kaplan-Meier methods and differences between the curves were tested using the log-rank method. A $P<0.05$ level (two sided) was adopted as the test for statistical significance. No subgroup analysis was prescheduled. Secondary outcome events and adverse events were analysed, and the intergroup comparisons were analyzed by using chi-square test.

\section{Results}

A total of 3505 patients from 88 clinical centers (hospitals) throughout China were recruited and randomly assigned. There were 1746 patients in the QSYQ group and 1759 in the aspirin group (Figure 2, according to CONSORT 2010). All randomised patients were included in ITT analyses and the 2956 patients who complied with the trial protocol were included in the PP analyses. 


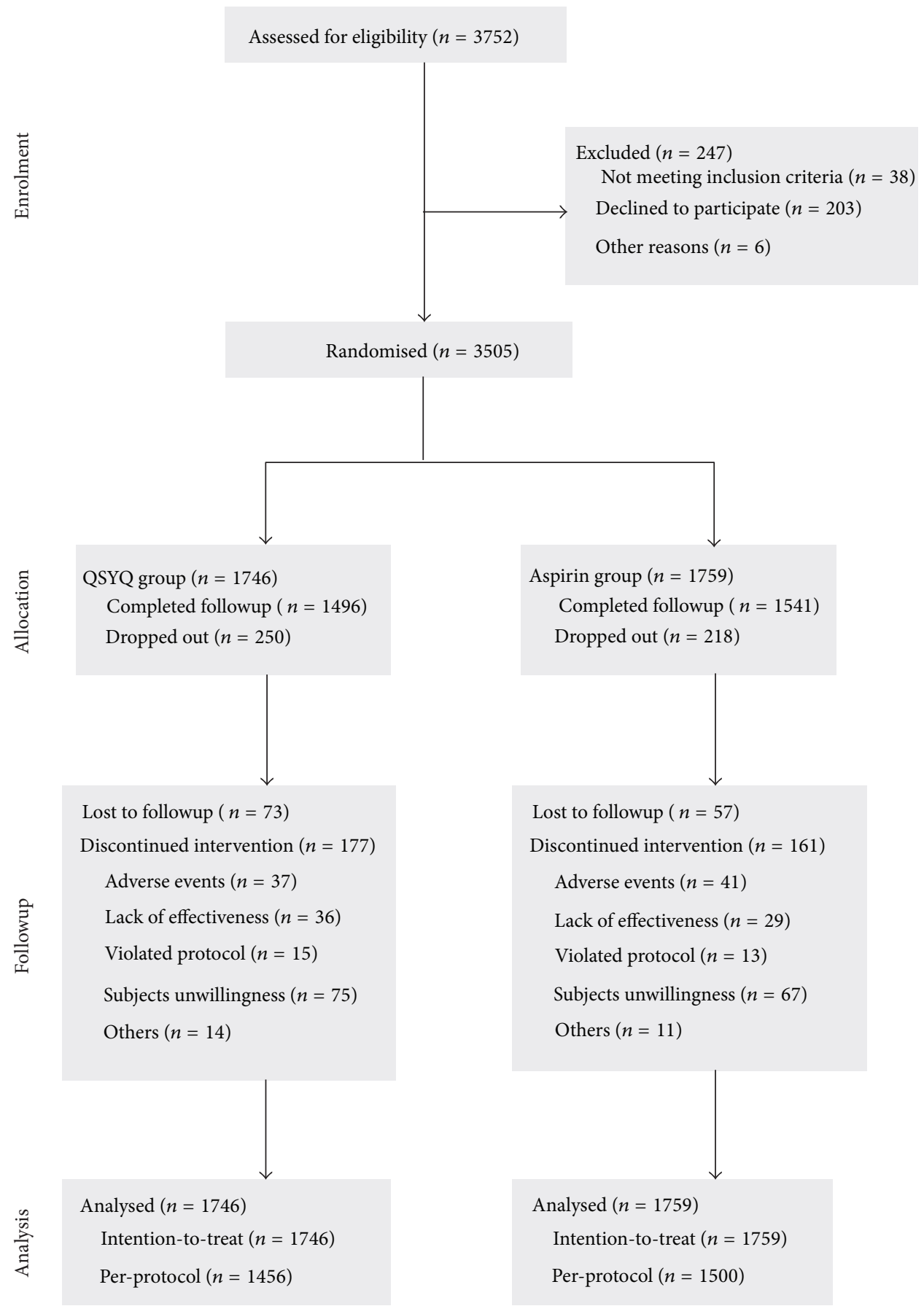

FIGURE 2: Flow diagram of participants through each stage of the trial.

3.1. Patients' Characteristics. Clinical characteristics of the included patients were shown in Table 1. In general, these baseline characteristics were similar in the two groups. The mean age of included patients was 58 years. Most of the patients were more than 12 months since their latest MI (3287 patients, 93.8\%). Most of the patients had an ST-sectionelevation MI, including 1109 (31.6\%) anterior MIs and 1558 $(44.45 \%)$ inferior MIs. About a third of patients had a body mass index (BMI) greater than $25 \mathrm{Kg} / \mathrm{m}^{2}$ and were considered to be overweight. About $70 \%$ of the participants were males. The aspirin group has a higher proportion of men than the QSYQ group which achieved statistical significance $(P=$ 0.027). This imbalance for gender also led to differences in the history of smoking $(P=0.048)$ and alcohol consumption $(P=0.036)$. Therefore, gender was adjusted for sensitivity analysis, by using Cox regression analysis. There were no 
TABLE 1: Baseline characteristics of included patients.

\begin{tabular}{|c|c|c|}
\hline Proportion & $\begin{array}{c}\text { QSYQ } \\
(n=1746)\end{array}$ & $\begin{array}{c}\text { Aspirin } \\
(n=1759)\end{array}$ \\
\hline \multicolumn{3}{|l|}{ Age at entry (years) } \\
\hline Mean (SD) & $58.35(9.02)$ & $58.28(8.99)$ \\
\hline$\leq 60$ & $910(52.12 \%)$ & $921(52.36 \%)$ \\
\hline$>60$ & $836(47.88 \%)$ & $838(47.64 \%)$ \\
\hline Gender-male* & $1191(68.21 \%)$ & $1260(71.63 \%)$ \\
\hline Smoking* & $773(44.27 \%)$ & $840(47.75 \%)$ \\
\hline Alcohol consumption* ${ }^{*}$ & $505(28.92 \%)$ & $568(32.29 \%)$ \\
\hline Nationality-Han & $1682(96.33 \%)$ & $1680(95.5 \%)$ \\
\hline \multicolumn{3}{|l|}{$\mathrm{BMI}\left(\mathrm{Kg} / \mathrm{m}^{2}\right)$} \\
\hline Mean (SD) & $24.24(2.82)$ & $24.28(2.86)$ \\
\hline$<25$ & $1125(64.43 \%)$ & $1122(63.79 \%)$ \\
\hline$\geq 25$ & $621(35.57 \%)$ & $637(36.21 \%)$ \\
\hline \multicolumn{3}{|l|}{ Time since latest MI (months) } \\
\hline$<6$ & $13(0.74 \%)$ & $12(0.68 \%)$ \\
\hline $6-12$ & $91(5.21 \%)$ & $102(5.80 \%)$ \\
\hline$>12$ & $1642(94.04 \%)$ & $1645(93.52 \%)$ \\
\hline \multicolumn{3}{|l|}{ Type of MI } \\
\hline Non-ST-elevation MI & $90(5.15 \%)$ & $69(3.92 \%)$ \\
\hline Mainly anterior MI & $529(30.30 \%)$ & $580(32.97 \%)$ \\
\hline Mainly inferior MI & $766(43.87 \%)$ & $792(45.03 \%)$ \\
\hline \multicolumn{3}{|l|}{ Medical history ${ }^{\#}$} \\
\hline Hyperlipidemia & $683(39.12 \%)$ & $680(38.66 \%)$ \\
\hline Hypertension & $753(43.13 \%)$ & $758(43.09 \%)$ \\
\hline Diabetes mellitus & $219(12.54 \%)$ & $227(12.91 \%)$ \\
\hline Stroke & $121(6.93 \%)$ & $106(6.03 \%)$ \\
\hline Gastritis & $227(13.00 \%)$ & $212(12.05 \%)$ \\
\hline \multicolumn{3}{|l|}{ Medications before entry ${ }^{\dagger}$} \\
\hline Anti-platelet agents & $1417(81.16 \%)$ & $1398(79.48 \%)$ \\
\hline Beta-blockers & $786(45.02 \%)$ & $800(45.48 \%)$ \\
\hline ACE inhibitors & $666(38.14 \%)$ & $669(38.03 \%)$ \\
\hline Lipid-lowering agents & $723(41.41 \%)$ & $705(40.08 \%)$ \\
\hline Calcium blockers & $238(13.63 \%)$ & $231(13.13 \%)$ \\
\hline Diuretics & $107(6.13 \%)$ & $90(5.12 \%)$ \\
\hline Cardiotonic agents & $40(2.29 \%)$ & $40(2.27 \%)$ \\
\hline Antiarrhythmia agents & $26(1.49 \%)$ & $29(1.65 \%)$ \\
\hline Nitrates & $1268(72.62 \%)$ & $1255(71.35 \%)$ \\
\hline Chinese patent medicines ${ }^{\S}$ & $620(35.51 \%)$ & $636(36.16 \%)$ \\
\hline
\end{tabular}

SD: standard deviation; MI: myocardial infarction; BMI: body mass index.

${ }^{*}$ The intergroup difference is statistically significant $(P<0.05)$.

"If a patient had more than one condition, they were counted for each of the different diseases.

${ }^{\dagger}$ If a patient took more than one drug, they were counted for each of the different drugs.

${ }^{\S}$ Chinese patent medicines were mainly used for heart disease.

inter-group differences in important prognostic factors such as medical histories, concomitant medications before entry, and medications during the treatment period.
3.2. Assessment of Patient Adherence. Of the included 3505 patients, 468 patients (218 in aspirin group and 250 in QSYQ group) without any recorded primary endpoint events did not complete their scheduled follow-up visits during the 12month treatment period (Figure 2). Fifteen patients in the QSYQ group and 10 patients in the aspirin group used antiplatelet drugs for more than one week in treatment period. The figures for prohibited Chinese herbal medicines were 33 in the QSYQ group and 28 in the aspirin group. These patients who withdrew during the treatment period or who took prohibited drugs were excluded from the PP analyses. Pill counting was used to assess patients' adherence to study prescriptions and revealed an average adherence of 95\% (95\% of the trial drugs were taken). This suggested that patients' adherence to the trial protocol was good.

3.3. Primary Outcomes. Table 2 presents the primary outcome (composite endpoints of cardiovascular death, nonfatal MI, and non-fatal stroke) at the two scheduled evaluation points: after completion of 12 months of treatment and at the 6 months followup following the end of study treatments. There are a total of 104 composite endpoints after 12-month treatment, including 51 cardiovascular deaths, 37 non-fatal MIs, and 16 non-fatal strokes. When the 6month posttreatment followup was completed, the composite endpoints increased to 131 cases, including 61 cardiovascular deaths, 52 non-fatal MIs, and 18 strokes. The 12-month and 18-month incidences of the primary outcome were $2.98 \%$ and $3.67 \%$ in the QSYQ group, respectively. The figures were $2.96 \%$ and $3.81 \%$ in the aspirin group. The differences between the two treatment groups were not statistically significant. Although minor changes to the results occurred after adjustment for gender, these changes did not make any meaningful difference to the results.

Cumulative analysis of the primary endpoints showed that the two curves were close during the 18-month followup (Figure 3). The hazard ratio (HR) for the primary outcome (QSYQ group versus aspirin group) was 0.977 (95\% CI: 0.694, 1.377; $P=0.895)$. The $\mathrm{PP}$ analysis generated a similar result (HR: 0.970; 95\% CI: 0.682, 1.379; $P=0.865$ ).

3.4. Secondary Outcomes. Secondary outcome measures were shown in Table 3. During the 12-month treatment, there were a total of $42(2.41 \%)$ secondary endpoints in the QSYQ group and 43 events (2.44\%) in the aspirin group. After the 18-month followup had been completed, these incidences of secondary endpoints increased to $2.98 \%$ and $3.30 \%$, respectively. There was no significant difference between the two groups at either of the two evaluation points.

3.5. Adverse Effects of Trial Drugs. Some potential adverse effects of aspirin and QSYQ were reported by patients as complaints or by the judgment of the investigator (Table 4). Nine patients in the aspirin group had a minor hemorrhage (two hemorrhagic strokes) compared to 2 patients (one hemorrhagic stroke) in the QSYQ group. There was no serious bleeding episode which required transfusion or caused death. Patients in the aspirin group had more gastric 
TABLE 2: Composite endpoints of cardiovascular death, nonfatal myocardial infarction, and nonfatal stroke (intention-to-be-treat analyses).

\begin{tabular}{|c|c|c|c|c|c|c|}
\hline Composite endpoints & QSYQ $(n=1746)$ & Aspirin $(n=1759)$ & HR $(95 \% \mathrm{CI})$ & $P$ value & $\begin{array}{l}\text { HR }(95 \% \text { CI }) \\
\text { Adjusted for } \\
\text { gender }\end{array}$ & $\begin{array}{c}P \text { value } \\
\text { Adjusted for } \\
\text { gender }\end{array}$ \\
\hline \multicolumn{7}{|c|}{ 12-month treatment completed } \\
\hline Composite endpoints & $52(2.98 \%)$ & $52(2.96 \%)$ & $1.02(0.69,1.50)$ & 0.928 & $1.03(0.70,1.52)$ & 0.872 \\
\hline Cardiovascular death & $28(1.60 \%)$ & $23(1.31 \%)$ & $1.24(0.71,2.15)$ & 0.450 & $1.26(0.73,2.19)$ & 0.411 \\
\hline Nonfatal MI & $18(1.03 \%)$ & $19(1.08 \%)$ & $0.97(0.51,1.84)$ & 0.917 & $0.98(0.51,1.87)$ & 0.952 \\
\hline Nonfatal stroke & $6(0.34 \%)$ & $10(0.57 \%)$ & $0.61(0.22,1.68)$ & 0.340 & $0.61(0.22,1.68)$ & 0.339 \\
\hline \multicolumn{7}{|c|}{ 6-month followup after study drugs terminated } \\
\hline Composite endpoints & $64(3.67 \%)$ & $67(3.81 \%)$ & $0.98(0.69,1.38)$ & 0.895 & $0.99(0.70,1.40)$ & 0.957 \\
\hline Cardiovascular death & $31(1.78 \%)$ & $30(1.71 \%)$ & $1.05(0.64,1.74)$ & 0.836 & $1.07(0.65,1.77)$ & 0.782 \\
\hline Nonfatal MI & $26(1.49 \%)$ & $26(1.48 \%)$ & $1.03(0.60,1.77)$ & 0.925 & $1.04(0.60,1.79)$ & 0.891 \\
\hline Nonfatal stroke & $7(0.40 \%)$ & $11(0.63 \%)$ & $0.65(0.25,1.68)$ & 0.373 & $0.65(0.25,1.68)$ & 0.377 \\
\hline
\end{tabular}

TABLE 3: Secondary outcome measure after 12-month treatment and 6-month followup after study drugs terminated.

\begin{tabular}{lcccccc}
\hline \multirow{2}{*}{ Secondary endpoints } & \multicolumn{3}{c}{12 -month treatment completed } & \multicolumn{3}{c}{ 18-month followup accomplished } \\
& QSYQ $(n=1746)$ & Aspirin $(n=1759)$ & HR $(95 \%$ CI $)$ & QSYQ $(n=1746)$ & Aspirin $(n=1759)$ & HR $(95 \%$ CI $)$ \\
\hline Revascularization & $21(1.20 \%)$ & $18(1.02 \%)$ & $1.18(0.63,2.20)$ & $23(1.32 \%)$ & $26(1.48 \%)$ & $0.89(051,1.56)$ \\
Aggravated heart failure & $20(1.15 \%)$ & $23(1.31 \%)$ & $0.88(0.48,1.59)$ & $28(1.60 \%)$ & $28(1.59 \%)$ & $1.01(0.60,1.69)$ \\
Cardiac shock & $1(0.06 \%)$ & $1(0.06 \%)$ & $1.01(0.06,16.09)$ & $1(0.06 \%)$ & $2(0.11 \%)$ & $0.50(0.05,5.55)$ \\
Deep vein thrombosis & $0(0)$ & $1(0.06 \%)$ & - & $0(0)$ & $2(0.11 \%)$ & - \\
\hline Sum up & $42(2.41 \%)$ & $43(2.44 \%)$ & $0.98(0.65,1.50)$ & $52(2.98 \%)$ & $58(3.30 \%)$ & $0.90(0.62,1.31)$ \\
\hline
\end{tabular}

TABLE 4: Adverse effects potentially associated with aspirin and Qi-Shen-Yi-Qi Dripping Pills.

\begin{tabular}{lccc}
\hline Adverse effects & QSYQ group $(n=1746)$ & Aspirin group $(n=1759)$ & $P$ value \\
\hline Hemorrhage & $2(0.11 \%)$ & $9(0.51 \%)$ & 0.06 \\
Intracranial bleeding & $1(0.06 \%)$ & $2(0.11 \%)$ & - \\
Gastrointestinal bleeding & $0(0)$ & $4(0.23 \%)$ & - \\
Subcutaneous hemorrhage & $1(0.06 \%)$ & $3(0.17 \%)$ & - \\
Stomach pain & $38(2.18 \%)$ & $37(2.1 \%)$ & 0.88 \\
Gastric acid reflux & $14(0.80 \%)$ & $33(1.88 \%)$ & 0.007 \\
Anaphylaxis & $11(0.63 \%)$ & $7(0.40 \%)$ & 0.34 \\
\hline
\end{tabular}

acid reflux (1.88\%) than those in the QSYQ group $(0.8 \%)$. This difference is statistically significant $(P=0.007)$. The incidences of stomach pain and merely allergic reactions were similar between the two groups. These data confirm that both study drugs are of good safety, and QSYQ appears to be slightly safer than aspirin.

\section{Discussion}

In this trial, QSYQ shows similar effects to aspirin for the prevention of recurrent vascular events in patient with a previous MI. The rate of composite endpoints (cardiovascular death, non-fatal MI, and non-fatal stroke) after 12-month treatment was $2.98 \%$ in QSYQ group and $2.96 \%$ in aspirin group. The incidence of serious vascular events of this trial is lower than previous studies of secondary prevention for MI [8], which may be due to several factors.

First, this study included low-risk patients compared to previous studies: patients who had experienced PCI and
CABG were excluded, and the time since latest MI was more than 12 months in most (93.8\%) of the patients in this trial. Thus, the risk of recurrent vascular events might be decreased. Second, although anti-platelet agents and "YiqiHuoxue" Chinese herbal medicines were prohibited during the trial, other drugs including beta-blockers, ACE inhibitors, lipid-lowing drugs, and drugs for hypertension and diabetes were allowed. Third, the follow-up period is short. The three factors are likely to be the main reasons for the low incidence of serious vascular events. In the future, a study with a more broadly inclusive eligibility criteria and longer study duration should be adopted.

Modern management of myocardial infarction is built on a high quality, clinical evidence base. Anti-platelet, betablockers, ACE inhibitors, and statins have been recommended for clinical practice based on the results of randomized clinical trials and their systematic review and have substantially reduced mortality and morbidity associated with MI. However, most of these conventional drugs are 


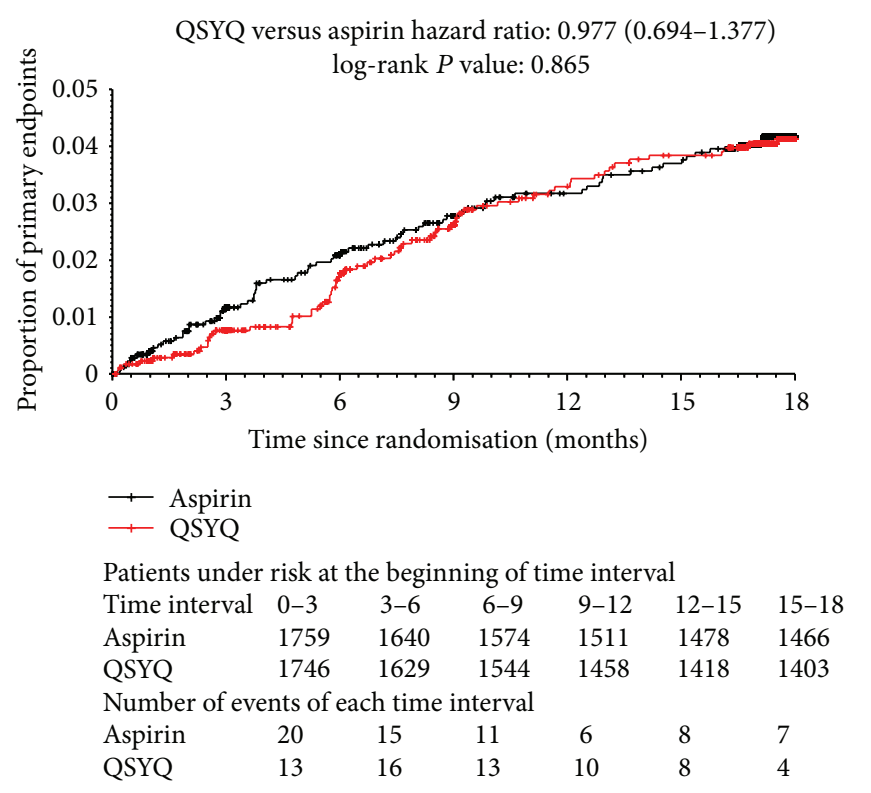

(a)

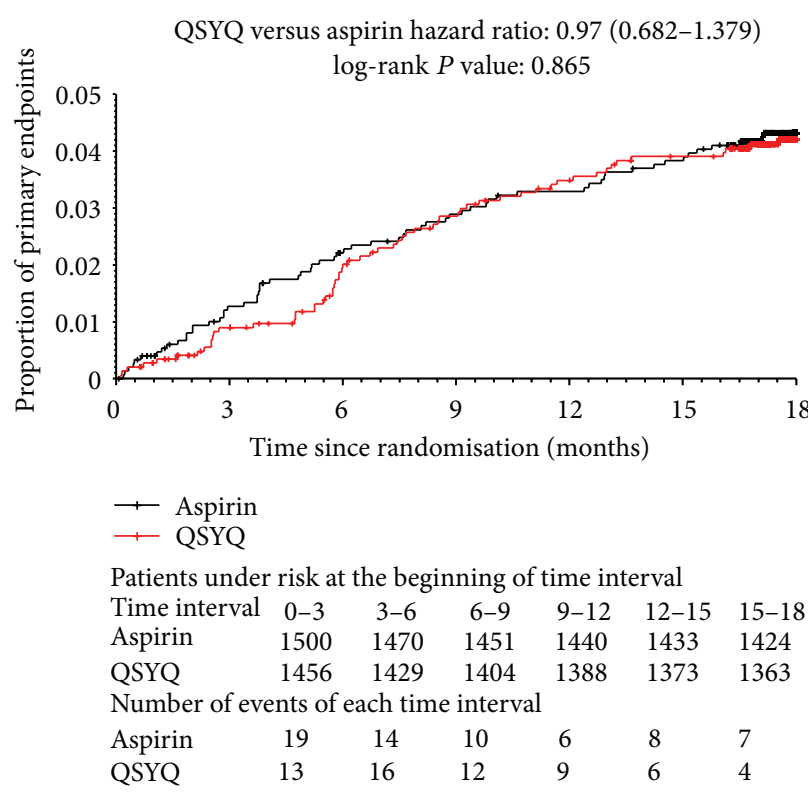

(b)

Figure 3: Cumulative incidence curves of the primary outcome composed of cardiovascular death, non-fatal myocardial infarction, and non-fatal stroke ((a) intention-to-treat analysis; (b) per-protocol analysis).

based on specific pathways, mainly single drug acting on single target $[6,20]$. This means that a patient might need to take several drugs concurrently, which leads to new problems, such as low adherence, high cost, and more adverse effects [21]. A new strategy for the management of patients following a myocardial infarction is needed.

The concept of a "polypill" was developed about 10 years ago, with a compound pill including several recommended drugs $[22,23]$. The Indian Polycap Study showed that a Polypill, composed of hydrochlorothiazide, atenolol, ramipril, simvastatin, and aspirin, had the desired effects and was safe as the individual pills [24]. Recently, a clinical trial in Sri Lanka, sponsored by the World Health Organization, has shown high acceptability of the Polypill to patients and physicians [25]. The Polypill is a new concept in western medicine but is not new in eastern medicine. QSYQ, which contains several kinds of active ingredient, is a classical polypill [26]. Over the past five years, in vivo and in vitro studies have revealed the integrated effects of QSYQ for MI, including protecting cardiac muscle cells [27], preventing cardiac ischemia-reperfusion injury via energy modulation [28], antagonizing ventricular remodeling [29], inhibiting inflammatory reaction and preventing the progress of atherosclerosis [30], and stabilizing atherosclerotic plaque by changing histological constitution [31]. A series of published studies suggested that QSYQ was a promising drug for secondary prevention of MI. However, the effects and mechanisms are not conclusive and need further studies to confirm and resolve uncertainties.

This study has some limitations. First, patients who had experienced PCI and CABG were excluded from this trial. This criterion limited the size of the sample and its representativeness. Second, biochemical measures (e.g., blood lipids) were not scheduled in this trial, in order to improve patient's adherence to followup. As a consequence, the effects of study drugs on these intermediate outcome measures were unknown. Third, our study was undertaken in China; whether the relative effects of the trial drugs would be similar in other ethnic groups is unknown.

Our study has several strengths. This is the first multicenter trial sponsored by the national funding of China and organized by researchers of Traditional Chinese Medicine to evaluate a Chinese patent medicine for the secondary prevention of MI. Randomization and blinding were well performed by using CRIVIS. Organization, execution, and evaluation were managed by different committees which worked independently.

\section{Conclusion}

This large sample clinical trial shows that QSYQ has similar effects to aspirin in the secondary prevention of $\mathrm{MI}$ and has fewer adverse effects. However, the low event rates of outcome measures were insufficient to generate a confirmatory conclusion. In addition, the polypill-like effect of QSYQ should be researched step by step. Further rigorously designed studies are warranted.

\section{Conflict of Interests}

There is no conflict of interests.

\section{Authors' Contribution}

All authors were involved in the trial design, the interpretation of the results, and in making revisions and corrections to the paper. The steering committee designed and revised 
the trial protocol. The coordinating center selected clinical centers and investigators and ensured that these departments worked together smoothly. The drug management center was in charge of producing, distributing, and monitoring study drugs and placebos. The clinical monitoring center checked the quality of trial documents. The endpoints committee verified all endpoints and adverse events, according to previously defined criteria. The biostatistics center was responsible for data analysis. The data management center was in charge of data capture, input, and preservation. MISPS collaborative group members are as follows. Steering committee-Tianjin University of Traditional Chinese Medicine (B. L. Zhang, X. M. Gao); Peking University First Hospital (C. Yao); The First Teaching Hospital of Tianjin University of TCM (J. P. Zhang, J. Y. Mao). Coordinating center-Tianjin University of Traditional Chinese Medicine (H. C. Shang, J. H. Zhang, H. Wang, H. J. Xu, W. K. Zheng, L. Zhang). Data management center-Chinese Academy of Traditional Chinese Medicine (B. Y. Liu, T. C. Wen). Biostatistics center-Peking University First Hospital (C. Yao, S. N. Zhu). Clinical monitoring centerXiyuang Hospital (W. L. Weng); Tianjin University of Traditional Chinese Medicine (H. B. Cao). End-points committeeThe Second Affiliated Hospital of Tianjin University of TCM (L. J. Sun). Drug management center-Tianjin University of Traditional Chinese Medicine (M. Ren).

\section{Acknowledgments}

The Ministry of Science and Technology of China (no. 2004BA716B01) and the State Administration of Traditional Chinese Medicine of China provided funding for this study (no. 200807004). They had no role in study design, data collection, data analysis, data interpretation, or writing of the report. The authors are grateful to the participants in the study and to the doctors, nurses, and administrative staff in the recruiting hospitals. Investigators who recruited at least 10 patients, by region and hospital, are as follows: Tianjin-The First Teaching Hospital of Tianjin University of TCM (J. Y. Mao, Z. Q. Zhao); The Second Affiliated Hospital of Tianjin University of TCM (W. X. Du, Y. Q. Zhao, T. Wang, C. Y. Liu); Tianjin Union Medicine Centre (K. Q. Liu, R. Wang); Tianjin Nankai Chinese Medicine Hospital (S. Gao, R. Y. Yuan); Tianjin WuQing Chinese Medicine Hospital (L. Q. Zhang, X. F. Liu); Tianjin Beichen Chinese Medicine Hospital (L. Shi); Tianjin Chinese Medicine Hospital (R. H. Fan, J. Zhang); The Second Hospital of Tianjin Medical University (L. F. Li, G. P. Li); Jixian People's Hospital (R. C. Xu, X. M. Zhou); Tianjin Dagang Hospital (X. Z. Zhou). Shandong-The Affiliated Hospital of Shandong University of TCM (H. J. Lin); Rizhao Hospital of TCM (X. G. Wang, S. M. Li); Qindao Hospital of TCM (L. B. Wei); Zibo Hospital of TCM (S. Q. Wang); Weifang Traditional Chinese Medicine Hospital (K. Q. Tang, K. G. Luan); The Affiliated Hospital of Medical College Qingdao University (Y. Li); Shandong Provincial Hospital (A. Y. Li, G. M. Si); Laiwu People's Hospital (Z. Y. Liang); Jinan Municipal Hospital of Traditional Chinese Medicine (H. Xu, X. J. Feng). Heilongjiang-First Affiliated Hospital of Heilongjiang University of Traditional Chinese Medicine
(Y. B. Zhou, B. Chen); Haerbin Hospital of Traditional Chinese Medicine (X. K. Liu, H. H. Wu); Qiqihaer Hospital of Traditional Chinese Medicine (B. Qi, J. Y. Li); The Third Affiliated Hospital of Qiqihaer Medical College (H. M. Liu, S. Sun); Jiamusi Hospital of Traditional Chinese Medicine (B. Sun); Mudanjiang Hospital of Traditional Chinese Medicine (S. K. Wang, X. F. Zhang). Beijing - Peking University First Hospital (X. M. Wang, G. X. Liu); Beijing Fu Wai Hospital (J. Z. Qu, L. H. Ma). Gansu-Gansu College of Traditional Chinese Medicine (Z. S. Jin, Y. K. Zhang); Gansu Province Hospital of Traditional Chinese Medicine (H. D. Deng, S. Zhou); Medical Department of Jiayuguan First Hospital; Wuwei People's Hospital (C. Y. Li, P. J. Wang); Tianshui Traditional Chinese Medical Hospital (J. C. Zhang, B. G. Cao); Jiuquan City People's Hospital (Y. F. Yuan); Jiayuguan City Jiugang Hospital (Y. J. Zhao); Gansu Province Cadres Medical Care Hospital (W. Huang, R. J. Yang); First Affiliated Hospital of Medical College of Shihezi University (W. G. Bian); Qingyang City People's Hospital (Z. Z. Liu, Y. F. Li); People's Hospital of Zhangye Municipality (G. Y. An, Z. Y. Yan). LiaoningThe Affiliated Hospital of Liaoning University of TCM (G. L. Yang, J. Zhang, F. R. Wang); The Second Affiliated Hospital of Liaoning University of TCM (B. Dong, D. H. Wang); Anshan Traditional Chinese Medical Hospital (J. T. Song, L. Cao); Dalian Traditional Chinese Medical Hospital (Y. L. Du, X. X. Yin); Dandong Traditional Chinese Medical Hospital (E. Wang, Z. R. Han). Jilin-The First Hospital of Jilin University (X. Z. Zhao); Meihekou Hospital of Traditional Chinese Medicine (Z. H. Cai, G. J. Wang). Xi'an-Xijing Hospital (Z. X. Dai, G. D. Shen); The 323 Liberation Army Hospital (H. M. Li); The 451 Liberation Army Hospital (B. X. Tuo, Y. Y. Che); Xi'an Jungong Hospital (B. X. Liu, L. K.). Shanxi-Shanxi Traditional Chinese Medical Hospital (T. F. Niu, H. X. Qi); Taiyuan City Centre Hospital (X. P. Chen); The Third Hospital of Shanxi Traditional Chinese Medical College (L. X. Ji, T. S. Zhang). Inner Mongolia-Inner Mongolia Medical College (X. P. Yang); Inner Mongolia Chinese-Mongolian Hospital (C. F. Liu, Y. Gao); Inner Mongolia Hospital (Z. Wu, A. G. Li); The Affiliated People's Hospital of Inner Mongolia Medical College (G. Liu, S. G. Zhang); Baogang Hospital (M. Q. Zhao, M. Sun). Hunan-Affiliated Hospital of Hunan Institute of Traditional Chinese Medicine (X. J. Hu, H. X. Zhu); The Fourth Hospital of Changsha (J. F. Li, Y. M. Yi); Wangyue Subdistrict Community Health Service Centre (S. H. Wen, L. Y. Liu). Hebei-Hebei Traditional Chinese Medical Hospital (Z. Q. Chen, H. W. Miao); Baiqiuen Peace Hospital (Z. B. Li); Shijiazhuang Traditional Chinese Medical Hospital (Z. Liu); Qinhuangdao Traditional Chinese Medical Hospital (L. R. Chen, H. Y. Wang). Henan-Nanyang Centre Hospital (B. Y. Mao); The First Nanyang People's Hospital (X. D. Zhu); Nanyang Traditional Chinese Medical Hospital (L. Q. Liu, D. X. Xie); Nanzhao Traditional Chinese Medical Hospital (Y. F. Zhang, J. C. Li); Henan Nanyang Zhangzhongjing Hospital (R. Ma). Anhui-Linquan Traditional Chinese Medical Institute (L. P. Jiang, Q. L. Zhou). Guangxi-Guangxi University of Traditional Chinese Medical (X. M. Fang); The First Affiliated Hospital of Guangxi Traditional Chinese Medical University (X. B. He); Ruikang Hospital of Guangxi Traditional Chinese Medical University (J. S. He, J. H. Yue). 


\section{References}

[1] World Health Organization, “The top ten causes of death," Fact sheet, no. 310, 2008.

[2] H. D. White and D. P. Chew, "Acute myocardial infarction," The Lancet, vol. 372, no. 9638, pp. 570-584, 2008.

[3] J. He, D. Gu, X. Wu et al., "Major causes of death among men and women in China," New England Journal of Medicine, vol. 353, no. 11, pp. 1124-1134, 2005.

[4] J. E. Sanderson, B. Mayosi, S. Yusuf et al., "Global burden of cardiovascular disease," Heart, vol. 93, no. 10, p. 1175, 2007.

[5] M. Moher, "Evidence of the effectiveness of interventions for secondary prevention and treatment of coronary heart disease in primary care-a review of the literature," NHS Executive, Anglia and Oxford Regional Health Authority, Oxford, UK, 1995.

[6] Y.-Z. Xiang, Y. Xia, X.-M. Gao, H.-C. Shang, L.-Y. Kang, and B.-L. Zhang, "Platelet activation, and antiplatelet targets and agents: current and novel strategies," Drugs, vol. 68, no. 12, pp. 1647-1664, 2008.

[7] C. Baigent, C. Sudlow, R. Collins, and R. Peto, "Collaborative meta-analysis of randomised trials of antiplatelet therapy for prevention of death, myocardial infarction, and stroke in high risk patients," British Medical Journal, vol. 324, no. 7329, pp. 7186, 2002.

[8] Antithrombotic Trialists' Collaboration, "Aspirin in the primary and secondary prevention of vascular disease: collaborative meta-analysis of individual participant data from randomised trials," The Lancet, vol. 373, no. 9678, pp. 1849-1860, 2009.

[9] L. A. García Rodríguez, S. Hernández-Díaz, and F. J. de Abajo, "Association between aspirin and upper gastrointestinal complications: systematic review of epidemiologic studies," British Journal of Clinical Pharmacology, vol. 52, pp. 563-571, 2001.

[10] S. Derry and Y. K. Loke, "Risk of gastrointestinal haemorrhage with long term use of aspirin: meta-analysis," British Medical Journal, vol. 321, no. 7270, pp. 1183-1187, 2000.

[11] G. De Berardis, G. Lucisano, A. D’Ettorre et al., "Association of aspirin use with major bleeding in patients with and without diabetes," Journal of the American Medical Association, vol. 307, no. 21, pp. 2286-2294, 2012.

[12] M. M. C. Hovens, J. D. Snoep, J. C. J. Eikenboom, J. G. van der Bom, B. J. A. Mertens, and M. V. Huisman, "Prevalence of persistent platelet reactivity despite use of aspirin: a systematic review," American Heart Journal, vol. 153, no. 2, pp. 175-181, 2007.

[13] J. D. Snoep, M. M. C. Hovens, J. C. J. Eikenboom, J. G. Van Der Bom, and M. V. Huisman, "Association of laboratory-defined aspirin resistance with a higher risk of recurrent cardiovascular events: a systematic review and meta-analysis," Archives of Internal Medicine, vol. 167, no. 15, pp. 1593-1599, 2007.

[14] G. Krasopoulos, S. J. Brister, W. S. Beattie, and M. R. Buchanan, "Aspirin "resistance" and risk of cardiovascular morbidity: systematic review and meta-analysis," British Medical Journal, vol. 336, no. 7637, pp. 195-198, 2008.

[15] Y.-Z. Xiang, L.-Y. Kang, X.-M. Gao, H.-C. Shang, J.-H. Zhang, and B.-L. Zhang, "Strategies for antiplatelet targets and agents," Thrombosis Research, vol. 123, no. 1, pp. 35-49, 2008.

[16] L. Yunfei, Q. Haibin, and C. Yiyu, "Identification of major constituents in the traditional Chinese medicine "QI-SHENYI-QI" dropping pill by high-performance liquid chromatography coupled with diode array detection-electrospray ionization tandem mass spectrometry," Journal of Pharmaceutical and Biomedical Analysis, vol. 47, no. 2, pp. 407-412, 2008.

[17] Y. Wang, J. Wang, L. Guo, and X. Gao, "Antiplatelet effects of qishen yiqi dropping pill in platelets aggregation in hyperlipidemic rabbits," Evidence-Based Complementary and Alternative Medicine, vol. 2012, Article ID 205451, 5 pages, 2012.

[18] J. Chen, D. Z. Qu, and R. Y. Wang, "Evaluation of Qishenyiqi Dripping Pills for aspirin resistance in patients with coronary heart disease," Chinese Traditional and Herbal Drugs, vol. 39, pp. 96-97, 2008.

[19] J. S. Alpert, K. Thygesen, E. Antman, and J. P. Bassand, "Myocardial infarction redefined-a consensus document of The Joint European Society of Cardiology/American College of Cardiology Committee for the redefinition of myocardial infarction," Journal of the American College of Cardiology, vol. 36, pp. 959-969, 2000.

[20] Y. Z. Xiang, H. C. Shang, and B. L. Zhang, "Secondary prevention of myocardial infarction and applicable prospect of traditional Chinese medicine," Medical Recapitulate, vol. 13, pp. 263-265, 2007.

[21] G. Sanz, V. Fuster, M.-J. Kravis, H. R. Kravis, and R. Gorlin, "Fixed-dose combination therapy and secondary cardiovascular prevention: rationale, selection of drugs and target population," Nature Clinical Practice Cardiovascular Medicine, vol. 6, no. 2, pp. 101-110, 2009.

[22] N. J. Wald and M. R. Law, "The Indian Polycap Study (TIPS)," The Lancet, vol. 374, p. 781, 2009.

[23] N. J. Wald and M. R. Law, "A strategy to reduce cardiovascular disease by more than $80 \%$," British Medical Journal, vol. 326, no. 7404, pp. 1419-1423, 2003.

[24] S. Yusuf, P. Pais, R. Afzal et al., "Effects of a polypill (Polycap) on risk factors in middle-aged individuals without cardiovascular disease (TIPS): a phase II, double-blind, randomised trial," The Lancet, vol. 373, pp. 1341-1351, 2009.

[25] E. Z. Soliman, S. Mendis, W. P. Dissanayake et al., "A Polypill for primary prevention of cardiovascular disease: a feasibility study of the World Health Organization," Trials, vol. 12, article 3, 2011.

[26] J. Gu, G. Yuan, Y. Zhu, and X. Xu, "Computational pharmacological studies on cardiovascular disease by Qishenyiqi Diwan," Science in China B, vol. 39, pp. 1415-1423, 2009.

[27] S. Q. Lin, X. H. Wei, P. Huang, Y. Y. Liu et al., "QiShenYiQi Pills prevents cardiac ischemia-reperfusion injury via energy modulation," International Journal of Cardiology, 2012.

[28] C. Hong, Y. Wang, J. Lou, Q. Liu, H. Qu, and Y. Cheng, "Analysis of myocardial proteomic alteration after Qishenyiqi formula treatment in acute infarcted rat hearts," Zhongguo Zhongyao Zazhi, vol. 34, no. 8, pp. 1018-1021, 2009.

[29] W. X. Du, M. D. Zhu, L. M. Feng et al., "Intervention effect of Qishenyiqi dripping pills on early ventricular remodeling after acute myocardial infarction," Chinese Journal of Evidence-Based Cardiovascular Medicine, vol. 28, pp. 41-43, 2008.

[30] F. F. Yan, Y. Liu, Y. F. Liu, and Y. X. Zhao, "Effects of Qishenyiqi Dripping pills on high sensitivity $\mathrm{C}$ reactive protein in experimental atherosclerosis rabbits," Shanghai Journal of Traditional Chinese Medicine, vol. 41, pp. 59-60, 2007.

[31] F. F. Yan, Y. Liu, Y. F. Liu, and Y. X. Zhao, "Effect of Qishenyiqi dripping pills on histology of experimental atherosclerotic plaque," Journal of Nanjing University of Traditional Chinese Medicine, vol. 23, pp. 295-297, 2007. 


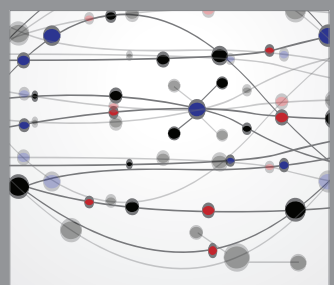

The Scientific World Journal
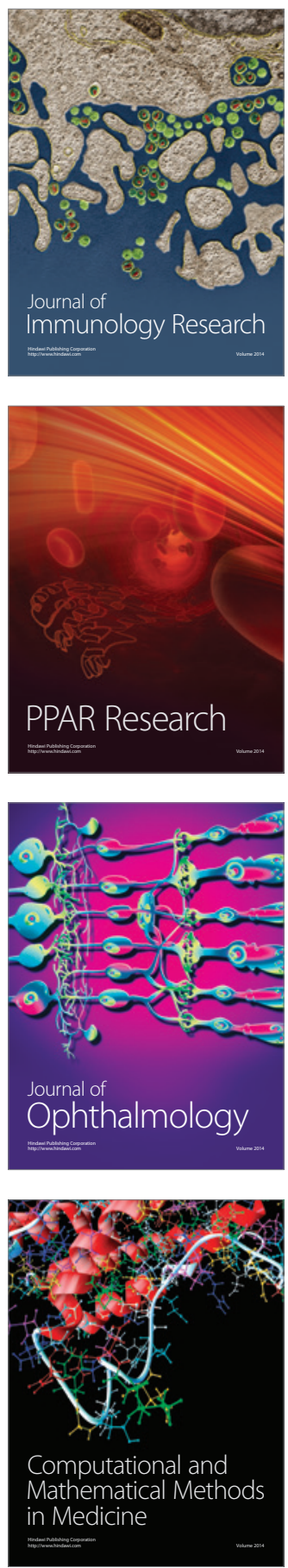

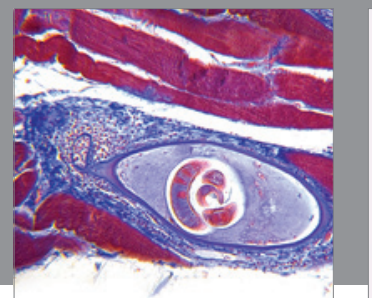

Gastroenterology

Research and Practice
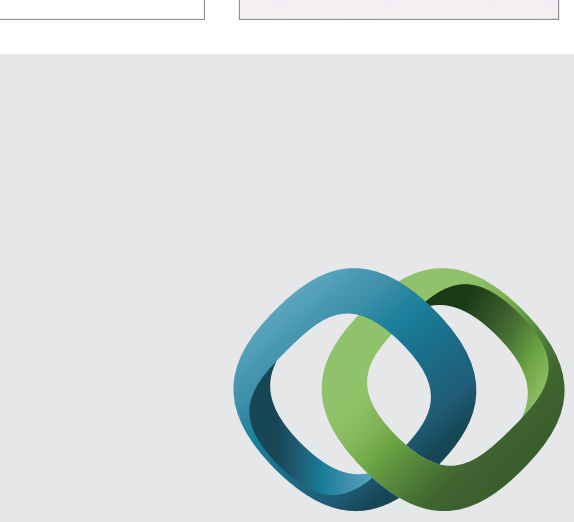

\section{Hindawi}

Submit your manuscripts at

http://www.hindawi.com
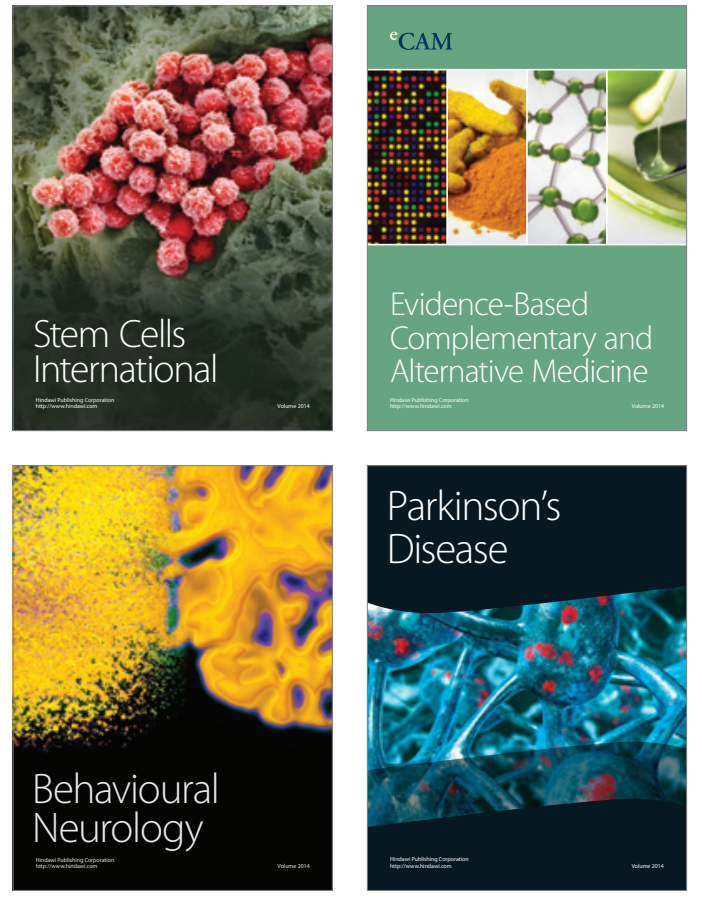
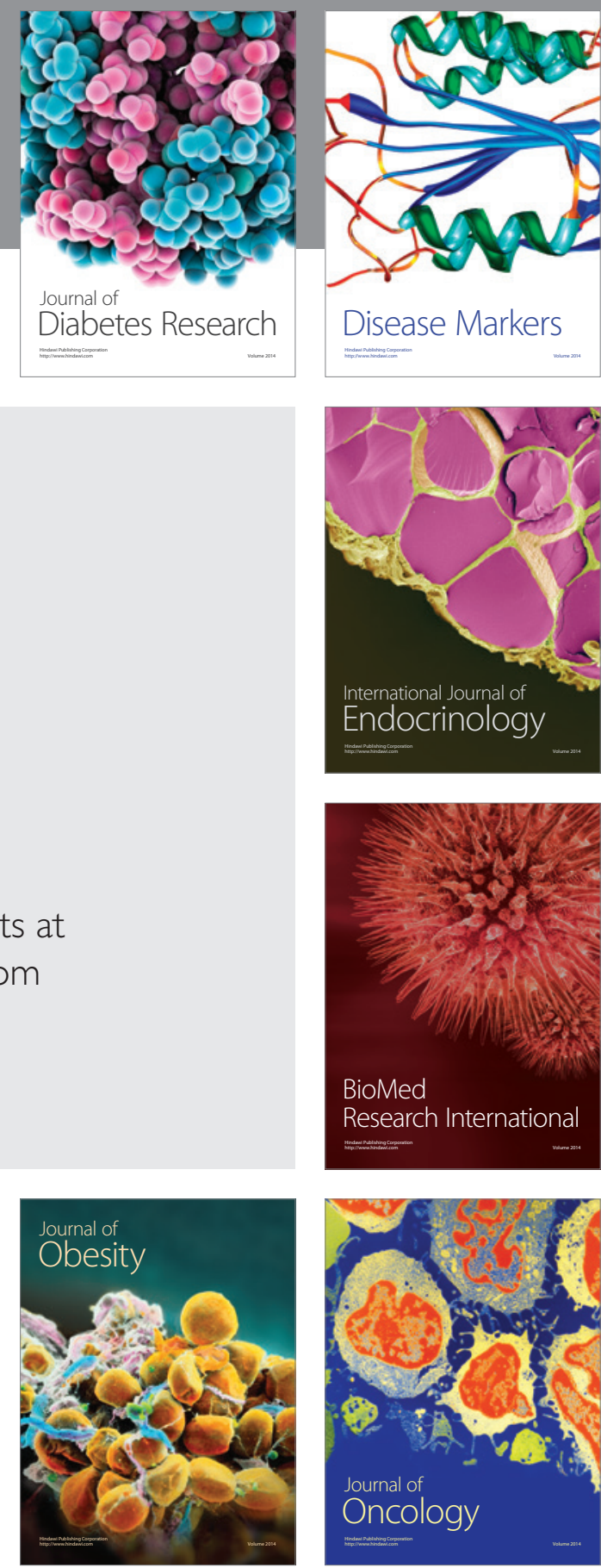

Disease Markers
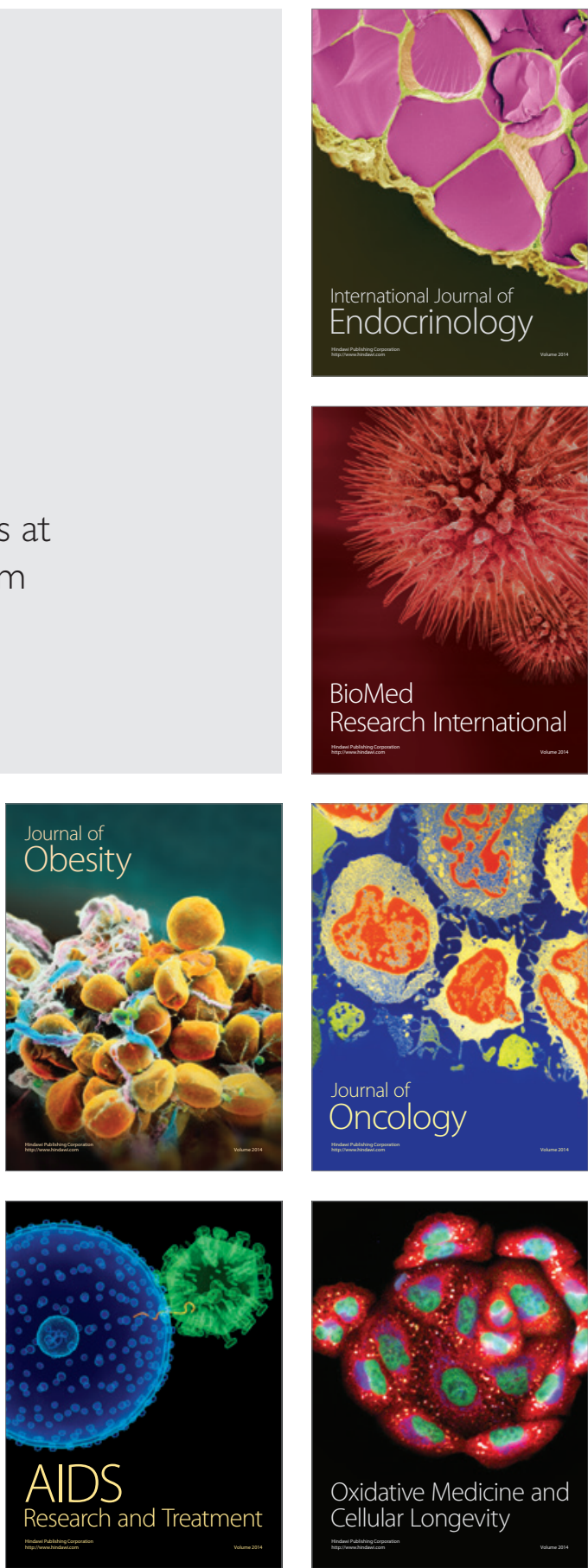